Article

\title{
Cloning and Functional Characterization of a Flavonoid Transport-Related MATE Gene in Asiatic Hybrid Lilies (Lilium spp.)
}

\author{
Hua Xu ${ }^{1,2}$, Panpan Yang ${ }^{1}$, Yuwei Cao ${ }^{1}$, Yuchao Tang ${ }^{1}$, Guoren He ${ }^{1}$, Leifeng Xu ${ }^{1, *(D)}$ and \\ Jun Ming ${ }^{1, *}$ \\ 1 Institute of Vegetables and Flowers, Chinese Academy of Agricultural Sciences, Beijing 100081, China; \\ ahxuhua@163.com (H.X.); yangpanpan1988@126.com (P.Y.); 13067739520@163.com (Y.C.); \\ tangyuchao100@126.com (Y.T.); hgr0222@sina.com (G.H.) \\ 2 College of Life Science, Gannan Normal University, Ganzhou 341000, China \\ * Correspondence: xuleifeng@caas.cn (L.X.); mingjun@caas.cn (J.M.)
}

Received: 20 March 2020; Accepted: 8 April 2020; Published: 12 April 2020

\begin{abstract}
Previous studies have suggested that multidrug and toxic compound extrusion (MATE) proteins might be involved in flavonoid transportation. However, whether MATE proteins are involved in anthocyanin accumulation in Lilium is unclear. Here, a flavonoid transport-related MATE candidate gene, LhDTX35, was cloned from the Asiatic hybrid lily cultivar 'Tiny Padhye' by rapid amplification of $5^{\prime}$ and $3^{\prime}$ cDNA ends (RACE) and found to encode 507 amino acids. BLASTx results indicated that LhDTX35 showed high homology to the DTX35 genes of other species. Bioinformatics analysis predicted that the protein encoded by LhDTX35 possessed 12 typical transmembrane segments and had functional domains typical of the MATE-like superfamily. Phylogenetic analysis grouped LhDTX35 in the same clade as the DTX35 of other species. Notably, the expression pattern of LhDTX35 was positively correlated with floral anthocyanin accumulation in 'Tiny Padhye'. A subcellular localization assay showed that the protein encoded by LhDTX35 was plasmalemma localized but not nuclear, indicating that the LhDTX35 gene may function as a carrier protein to transport anthocyanins in Lilium. Functional complementation of the Arabidopsis DTX35 gene demonstrated that LhDTX35 could restore silique-infertility and the anthocyaninless phenotype of an Arabidopsis DTX35 mutant. These results indicated that LhDTX35 might be involved in anthocyanin accumulation in Lilium.
\end{abstract}

Keywords: Lilium; anthocyanins; transportation; MATE; LhDTX35

\section{Introduction}

Lilies (Lilium spp.) have high commercial and ornamental value due to their various floral colors and coloration patterns. Anthocyanins are one of the major pigments affecting the coloration of the floral tepals in Lilium spp. [1-4]. Anthocyanins are synthesized in the cytosol but their transfer to the vacuole is necessary for plant tissues to exhibit brilliant colors $[5,6]$. The mechanism of anthocyanin transfer is still unclear, although anthocyanin biosynthesis at the molecular level has been studied extensively leading to the discovery of enzyme-coding structural and regulatory genes related to anthocyanin biosynthesis [7,8]. To date, glutathione S-transferases (GSTs), multidrug and toxic compound extrusion (MATE) proteins, and the ATP-binding cassette (ABC) transporter have been proposed to be involved in anthocyanin transportation in many species [9-11].

MATE transporters perform various transport functions in plants, including the transportation of anthocyanin [12]. A MATE transporter related to flavonoid accumulation was isolated for the first time during screening of a TT12 Arabidopsis mutant with altered seed coloration [13]. Subsequently, 
orthologs of AtTT12 were obtained from many other species, such as upland cotton, blueberry, Medicago truncatula, apple, and grapevine, and orthologs of the TT12 gene were verified to be related to flavonoid transportation [6,14-17]. To date, the enzyme-coding structural genes and regulatory genes of anthocyanin biosynthesis in Lilium have been characterized thoroughly [2,4,18-20]; however, whether MATE transport is involved in anthocyanin transportation in Lilium remains unclear.

In this study, based on our RNA-seq data published previously [21], we isolated a flavonoid transport-related MATE gene, LhDTX35, from Lilium 'Tiny Padhye'. Then, expression profiling, identification, and functional analysis of this gene in anthocyanin transportation were performed using 'Tiny Padhye' as a subject. This study demonstrates that a gene encoding MATE is critical for anthocyanin accumulation in Lilium, and promotes an understanding of the mechanism underlying MATE involvement in anthocyanin transportation in Lilium.

\section{Materials and Methods}

\subsection{Plant Material}

The Asiatic lily cultivar 'Tiny Padhye' was grown in a greenhouse $\left(25^{\circ} \mathrm{C}\right.$, relative humidity $60 \%-70 \%, 16 \mathrm{~h} / 8 \mathrm{~h}$ light/dark cycles) at the Chinese Academy of Agricultural Sciences (Beijing, China). The upper parts and bases of the inner tepals were collected at four different developmental stages. The tepal developmental stages were defined as described in $\mathrm{Xu}$ LF et al. [21]: Stage 1 (S1: no anthocyanin pigment is visible in tepals), stage 2 (S2: anthocyanin pigment becomes visible on tepals), stage 3 (S3: the day before anthesis, lower halves of tepals are fully pigmented), and stage 4 (S4: the first day after anthesis) (Figure 1). Samples were obtained from 15 flowers and pooled together as one biological sample; three independent biological replicates were collected for each stage.

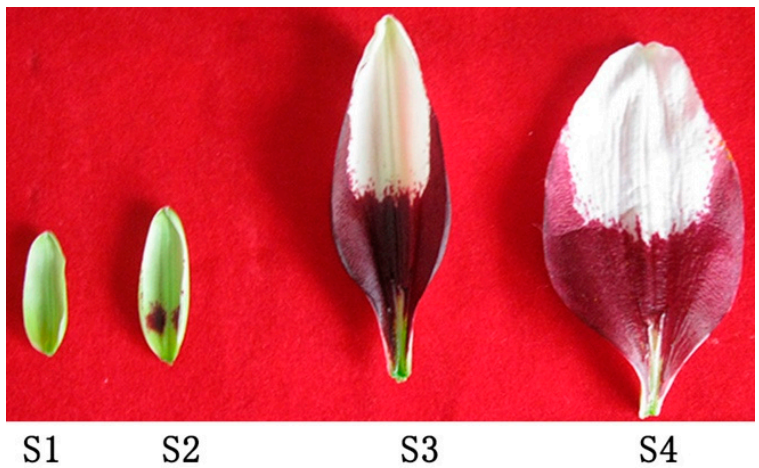

Figure 1. Tepals of the Asiatic lily cultivar 'Tiny Padhye' at four different developmental stages.

Nicotiana tabacum was also grown in a greenhouse, as described above for environmental conditions. Seedlings at the four-true-leaf stage (6-week-old) were used for the subcellular localization experiment.

\subsection{Isolation of a MATE-Like Gene}

Six primers (LhMATE-YZ-F1, LhMATE-YZ-R1, LhMATE-3'RACE-GSP, LhMATE-3'RACE-nest, LhMATE-5'RACE, and LhMATE-5'RACE-nest) were designed based on unigenes encoding MATE-like proteins from our previous RNA-seq experiment (Table 1). First-strand cDNA was cloned using RNA isolated from the colored parts of the tepal at stage 2 as a template, according to the RACE kit manufacturer's instructions (Takara, Dalian China). To obtain the full-length MATE cDNA, the 5'-end and 3 '-end sequences of the identified gene were identified using stage 2 total RNA from the basal (colored) tepal regions and a RACE kit, according to the recommendations of the manufacturer (Takara, Dalian, China). Five independent RACE cDNA clones were sequenced by Sangon Biotech (Shanghai, China). Based on the 5'-RACE and 3'-RACE sequencing results, the primers LhDTX35-F 
and LhDTX35-R (Table 1) were designed to amplify the entire gene region containing the open reading frame (ORF) from the first-strand cDNA.

Table 1. All primers used in this study.

\begin{tabular}{cc}
\hline Primers & Primer Sequence $^{\left(\mathbf{5}^{\prime} \mathbf{- 3}^{\prime} \mathbf{)}\right.}$ \\
\hline LhMATE-YZ-F1 & GTTGCGGTTATCACATCCCT \\
LhMATE-YZ-R1 & CTTCTTCCCCTCACAGTCTA \\
LhDTX35-3'RACE-GSP & CGGTGGGTGGCAAGGTCTGGTAGC \\
LhDTX35-3'RACE-nest & GGGCTATCCGTTACATTTGGGTGTGC \\
LhDTX35-5'RACE, & CCAAATCCCCTGCACACCCAAA \\
LhDTX35-5'RACE-nest & TGGTTCATTCTGTCCACCCCAC \\
LhDTX35-F, & AGTGGGAGAGAGAGAGAGGCGA \\
LhDTX35-R & CTTCTTCCCCTCACAGTCTATGC \\
LhDTX35-YG-F & CCAAGAGGATGACATTGCCGAG \\
LhDTX35-YG-R & TGGAGGATGAGGGTGAAGAAGC \\
LhDTX35-DW-F & CCCCCGGGATGGAAGATCCGCTTCTGAGAC \\
LhDTX35-DW-R & GCTCTAGACACTAACTTGACTTTGTTGGTT \\
DW-YZ-F & CGGGCTGTTGCGAAAATA \\
DW-YZ-R & TGCCGTTCTTCTGCTTGTC \\
LhACT-YG-F & GCATCACACCTTCTACAACG \\
LhACT-YG-R & GAAGAGCATAACCCTCATAGA \\
Actin-F & CGTGACCTTACTGATTACCT \\
Actin-R & AGCGATACCTGAGAACATAG \\
LhDTX35-noci-F & CGGGGGACTCTTGACCATGGAAGATCCGCTTCTGAGACAT \\
LhDTX35-Bahm-R & GGAAATTCGAGCTGGTCACCTGTAATCTAACTTGACTTTGTTGGTT \\
LhDTX35-YZ-F & CGGGCTGTTGCGAAAATA \\
LhDTX35-YZ-R & TGCCGTTCTTCTGCTTGTC \\
\hline
\end{tabular}

\subsection{Sequence Bioinformatics Analysis}

A BLASTx search of MATE-like gene ORF sequences was conducted to analyze sequence similarity. Protein conserved domain prediction was performed by NCBI conserved domain online software [22]. The protein transmembrane domains were analyzed by TMHMM Server V.2.0 [23].

\subsection{Phylogenetic Analysis}

The amino acid sequences of genes encoding MATEs from different plants were used for phylogenetic analysis. Sequence alignment was performed using CLUSTAL X. Based on this alignment, a Neighbor-Joining (NJ) tree was constructed using MEGA (version 7.0). Bootstrap values were calculated using 1000 replicate analyses.

\subsection{Gene Expression Analysis}

Quantitative real-time polymerase chain reaction (qRT-PCR) was performed using SYBR Premix Ex Taq (Takara, Dalian, China). The primers used to amplify the MATE-like gene segment are shown in Table 1. The reaction parameters were as follows: (1) $95^{\circ} \mathrm{C}$ for $1 \mathrm{~min}$; (2) 40 cycles of $95^{\circ} \mathrm{C} \mathrm{for} 20 \mathrm{~s}$, $60{ }^{\circ} \mathrm{C}$ for $10 \mathrm{~s}$, and $72{ }^{\circ} \mathrm{C}$ for $25 \mathrm{~s}$; and (3) a melt curve program $\left(65^{\circ} \mathrm{C}\right.$ to $95{ }^{\circ} \mathrm{C}$ with an increment in temperature of $0.5^{\circ} \mathrm{C}$ every $0.05 \mathrm{~s}$ ). The signal was monitored using a CFX96 real-time system (Bio-Rad, CA, USA). The average Cq value was calculated from three biological and three technical replicates. To normalize the differences in the amounts of mRNA from other genes, the amount of Lhactin mRNA was determined for each sample and the relative expression level of LhDTX35 was analyzed using the $2^{-\triangle \Delta C T}$ method [24]. The error bars represented the \pm SEs from three independent experiments. The data were analyzed by ANOVA using SAS software. 


\subsection{Subcellular Localization Analysis}

Using the transient expression vector pCAMBIA2300-35s-GFP, the subcellular localization of the LhDTX35 gene was analyzed. To construct the expression vector 35S::LhDTX35-GFP, primers containing the $\mathrm{XbaI}$ and BamHI sites were designed to amplify the LhDTX35 gene ORF domain using the primer LhDTX35-DW-F/R (Table 1). The PCR products were separated on a 1\% agarose gel and then purified. The empty vector pCAMBIA2300-35s-GFP was digested with the restriction enzymes XbaI and BamHI according to the manufacturer's instructions (Thermo Scientific FastDigest, America). Subsequently, the purified PCR product and cleaved empty vector were fused using T4 DNA Ligase (TransGen, China). Positive clones were selected and confirmed by sequencing and then transformed into Escherichia coli DH5 $\alpha$ cells (TransGen, China). The sequencing primers (DW-YZ-F/R) are shown in Table 1. Finally, the plasmids pCAMBIA2300-GFP and pCAMBIA2300-GFP-LhDTX35 were each transformed into Agrobacterium tumefaciens (LBA4404) using the freeze-thaw method, as previously reported [25].

When N. tabacum had developed four true leaves, the underside of the leaves was infiltrated with Agrobacterium inocula using a $1 \mathrm{~mL}$ needleless syringe. The inoculated plants were grown in a phytotron for $24 \mathrm{~h}$ in the dark $\left(25^{\circ} \mathrm{C}\right.$, relative humidity $\left.60 \%-70 \%\right)$. Then, the plants were grown under $16 \mathrm{~h} / 8 \mathrm{~h}$ light/dark cycles for 5 days. Subsequently, the LhDTX35-GFP fusion protein in the N. tabacum epidermis cells was microscopically detected using a Leica confocal laser scanning microscope. The samples were illuminated with an argon ion laser using $488 \mathrm{~nm}$ light for GFP and a green HeNe laser using $543 \mathrm{~nm}$ light for chlorophyll autofluorescence.

\subsection{Complementation Analysis}

The 35S::LhDTX35 vector was constructed by replacing the GUS gene in the pCAMBIA3301 vector with the coding sequence of LhDTX35. The LhDTX35 ORF was amplified by PCR with primers LhDTX35-noci-F and LhDTX35-Bahm-R (Table 1). The LhDTX35 ORF fragments were cloned into the linearized vector pCAMBIA3301 and digested with the NocII and HindII restriction enzymes using a one-step seamless cloning kit (Transgen, Beijing, China). The binary vectors were introduced into A. tumefaciens GV3101 and subsequently used in Arabidopsis ttDTX35 mutant transformation with the floral dip method [26]. Seeds of the Arabidopsis LhDTX35 mutant, transgenic lines, and wild-type Arabidopsis were germinated and grown on Murashige \& Skoog Basic Medium 1/2 Macro (1/2 MS) medium. Transgenic plants were screened on $1 / 2 \mathrm{MS}$ medium plates that contained $50 \mathrm{mg} / \mathrm{L}$ kanamycin.

The expression of LhDTX35 was analyzed by RT-PCR with primers LhDTX35-YZ-F/R, and Arabidopsis Actin gene was control with primers Actin-F/R (Table 1).

\section{Results}

\subsection{Cloning and Sequence Analysis of the LhDTX35 Gene}

The full-length cDNA sequence of LhMATE-like was obtained by RACE and deposited in GenBank with the accession number MT001433. This sequence contained $1948 \mathrm{bp}$ and encoded 507 amino acids, as predicted by the NCBI ORF Finder tool (Figure 2). BLASTx results showed high homology to the DTX35 genes of other species, such as Dendrobium catenatum, Elaeis guineensis, Phoenix dactylifera, and Oryza sativa Japonica Group (ca. 71\%-76.36\% identity at the amino acid level). Therefore, the LhMATE-like cDNA isolated in this study was named LhDTX35. Conserved domain prediction revealed that LhDTX35 encoded functional domains typical of the MATE-like superfamily between 258 bp and $1562 \mathrm{bp}$ (Figure 3). Transmembrane domain analysis predicted that the protein encoded by LhDTX35 was a membrane protein with twelve typical transmembrane domains (Figure 4). A phylogenetic analysis revealed that LhDTX35 was closely related to the DTX35 protein in Dendrobium catenatum, sharing $76.36 \%$ amino acid identity (Figure 5). In conclusion, the isolated LhDTX35 gene was predicted to encode a membrane protein belonging to the MATE-like superfamily. 


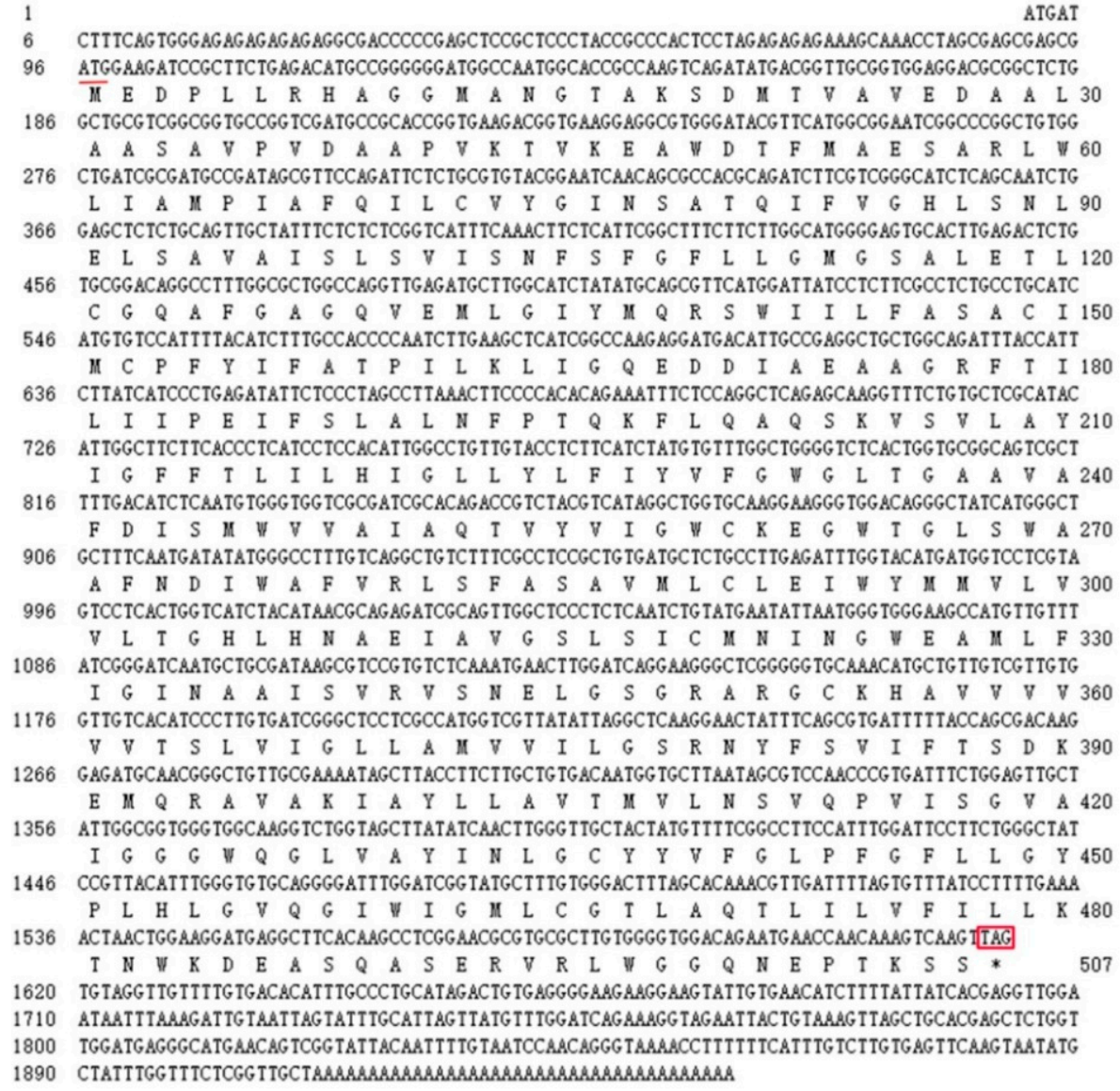

Figure 2. Nucleotide and deduced amino acid sequences of the LhDTX35 gene. The initiation codon is underlined, while the stop codon is labeled with an asterisk and box.

Conserved domains on [lcl|seqsig_ATGAT_9b1231ec6c3ce1847a57547d90eead08] View Concise Results . (리 Local query sequence

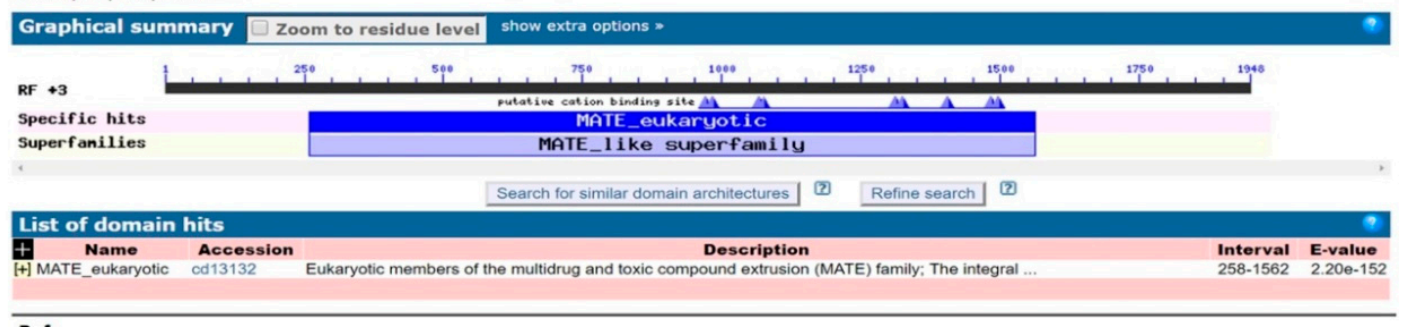

Figure 3. Conserved domain prediction for the protein encoded by LhDTX35.

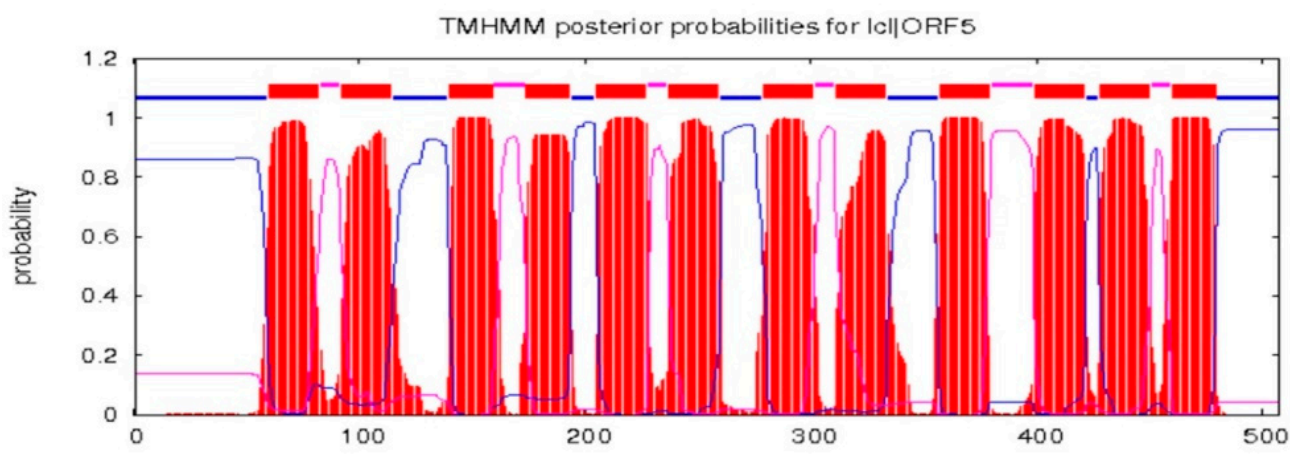

Figure 4. Transmembrane prediction for the protein encoded by LhDTX35. 


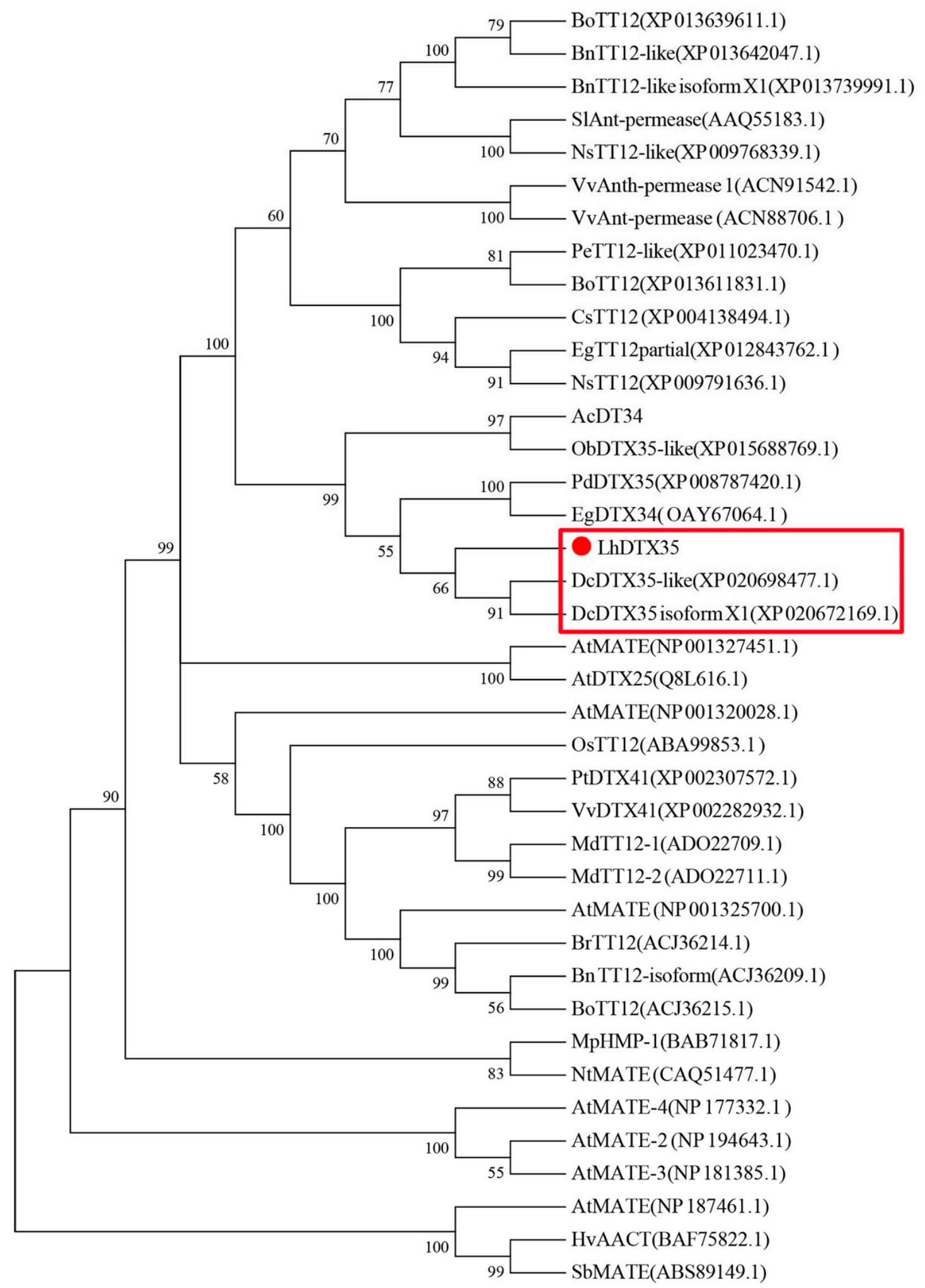

Figure 5. Phylogenetic tree of the predicted amino acid sequences of the Lilium multidrug and toxic compound extrusion (MATE) protein and MATE proteins from other plants. The phylogenetic tree was constructed using MEGA 6.0 software and the NJ method. The red dot indicates the Lilium MATE-like protein identified in this study. 


\subsection{Expression Analysis of LhDTX35}

Anthocyanin accumulated in only the basal tepal regions of 'Tiny Padhye'. We previously showed that anthocyanin content increased gradually during tepal development (Figure 6) [21]. Anthocyanin accumulation was the highest in tepals at stage 3 (Figure 6) [21]. To exploit whether the LhDTX35 gene was involved in anthocyanin accumulation in Lilium, qRT-PCR was performed using the upper and basal tepal regions at stages 1-4. These results showed that LhDTX35 gene expression was positively correlated with anthocyanin accumulation, which occurred in the bases of the tepals (Figure 7).

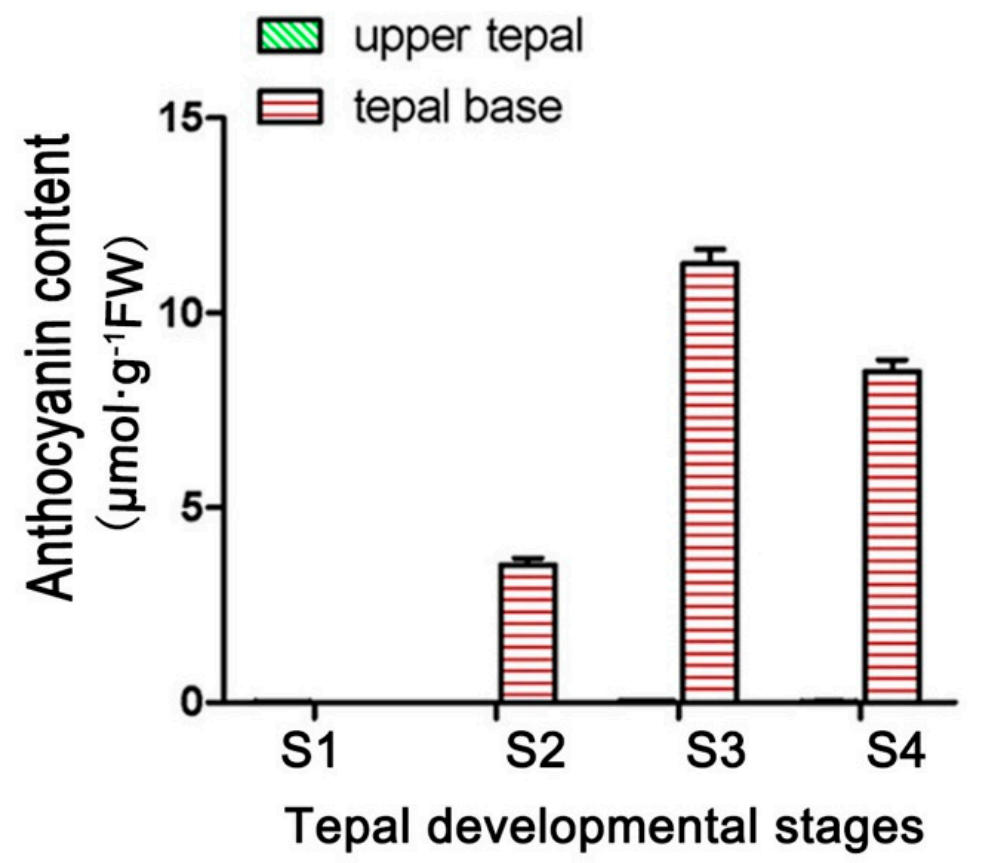

Figure 6. Changes in anthocyanin content at different developmental stages in Lilium [21].
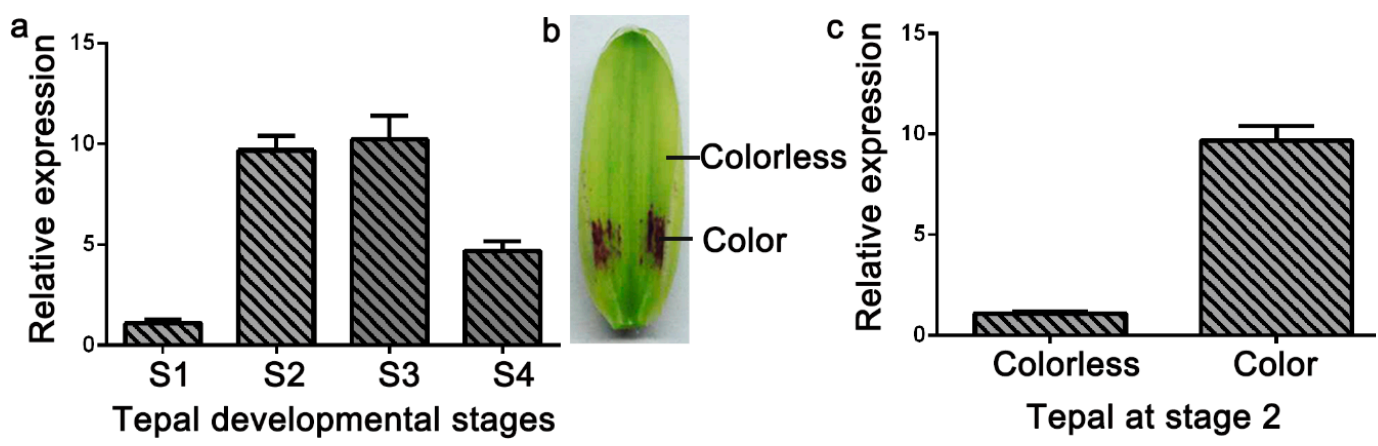

Figure 7. Spatiotemporal analysis of LhDTX35 gene expression at different stages of tepal development in Lilium 'Tiny Padhye'. (a) Expression profiles of LhDTX35 at stages 1-4 in the basal tepal region in Lilium 'Tiny Padhye'; (b) tepal at stage 2; (c) expression profiles of LhDTX35 at stage 2 between the upper and basal tepal regions in Lilium 'Tiny Padhye'.

\subsection{Subcellular Localization of LhDTX35}

A subcellular localization experiment was performed to further identify whether the protein encoded by the LhDTX35 gene was a membrane protein. The results showed that GFP fluorescence was distributed in the cytoplasm of $N$. tabacum leaf epidermal cells treated with the empty GFP vector (Figure 8a,b). In contrast, GFP fluorescence was located in the membrane of $N$. tabacum leaf epidermis 
cells treated with the LhDTX35 and GFP fusion protein vector (Figure $8 \mathrm{c}, \mathrm{d}$ ). These results indicated that the LhDTX35 gene encoded a membrane protein.
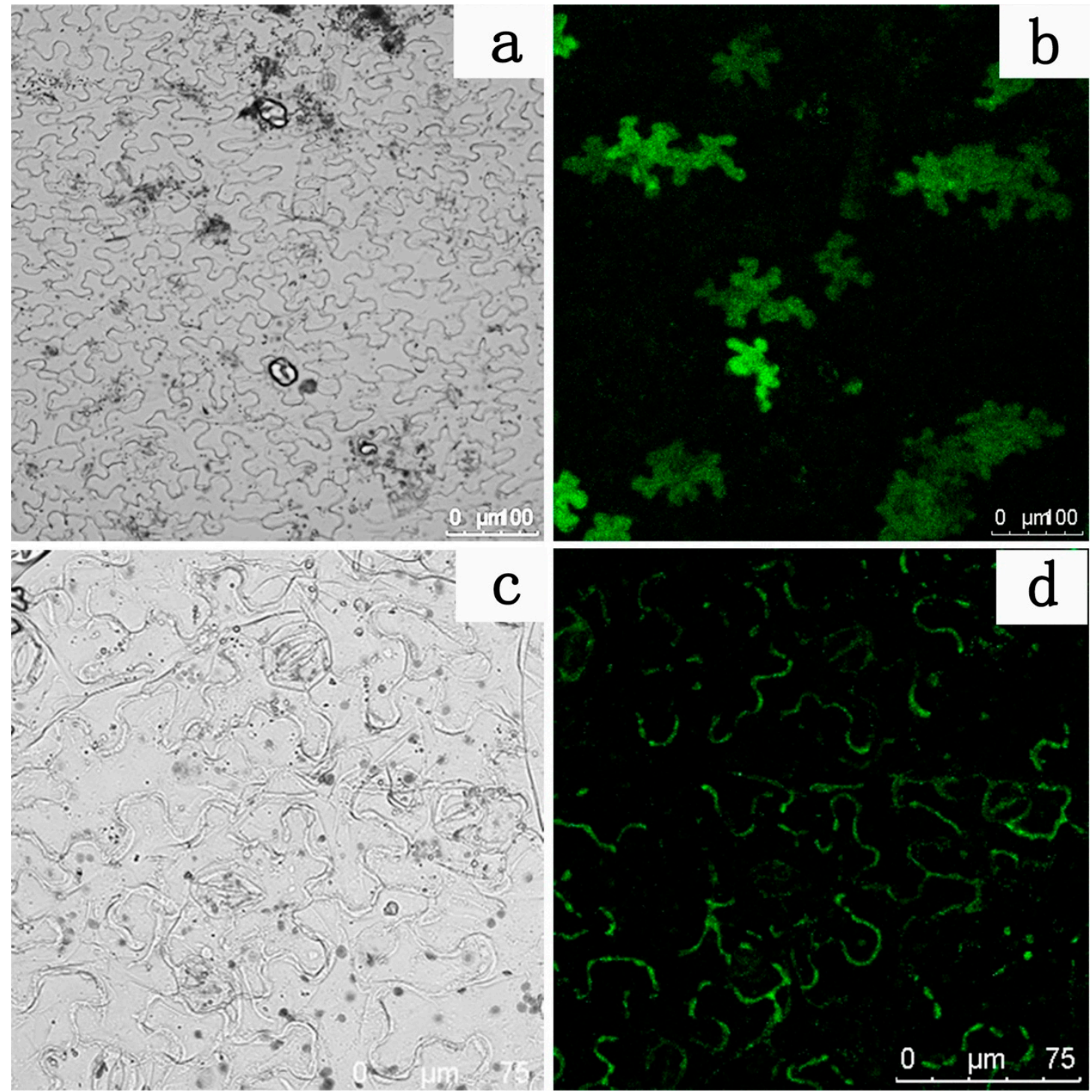

Figure 8. Subcellular localization of the LhDTX35-GFP fusion protein. (a,c) Bright field images; $(\mathbf{b}, \mathbf{d})$ GFP fluorescence images; (a,b) cells expressing the LhDTX35-GFP fusion protein; (c,d) cells expressing 35S::GFP as a control.

\subsection{Functional Analysis of the LhDTX35 Gene in the Arabidopsis DTX35 Gene Mutant}

An Arabidopsis genetic mutant lacking DTX35 was selected to investigate the functionality of the LhDTX35 gene. The coding sequence of the LhDTX35 gene was transferred into the Arabidopsis DTX35 mutant under the control of the cauliflower mosaic virus (CaMV) 35S promoter. The hypocotyls of wild-type plants and transgenic lines expressing LhDTX35 were red. In contrast, the hypocotyls of the Arabidopsis DTX35 mutant were green, except for the junctions between the cotyledons (Figure 9B). The silique fertility of the Arabidopsis DTX35 mutant was low but normal fertility was restored in the complementation experiment (Figure 9C). These results showed that the function of the LhDTX35 gene was similar to that of the DTX35 gene in Arabidopsis. Furthermore, the LhDTX35 gene may be involved in anthocyanin accumulation. 


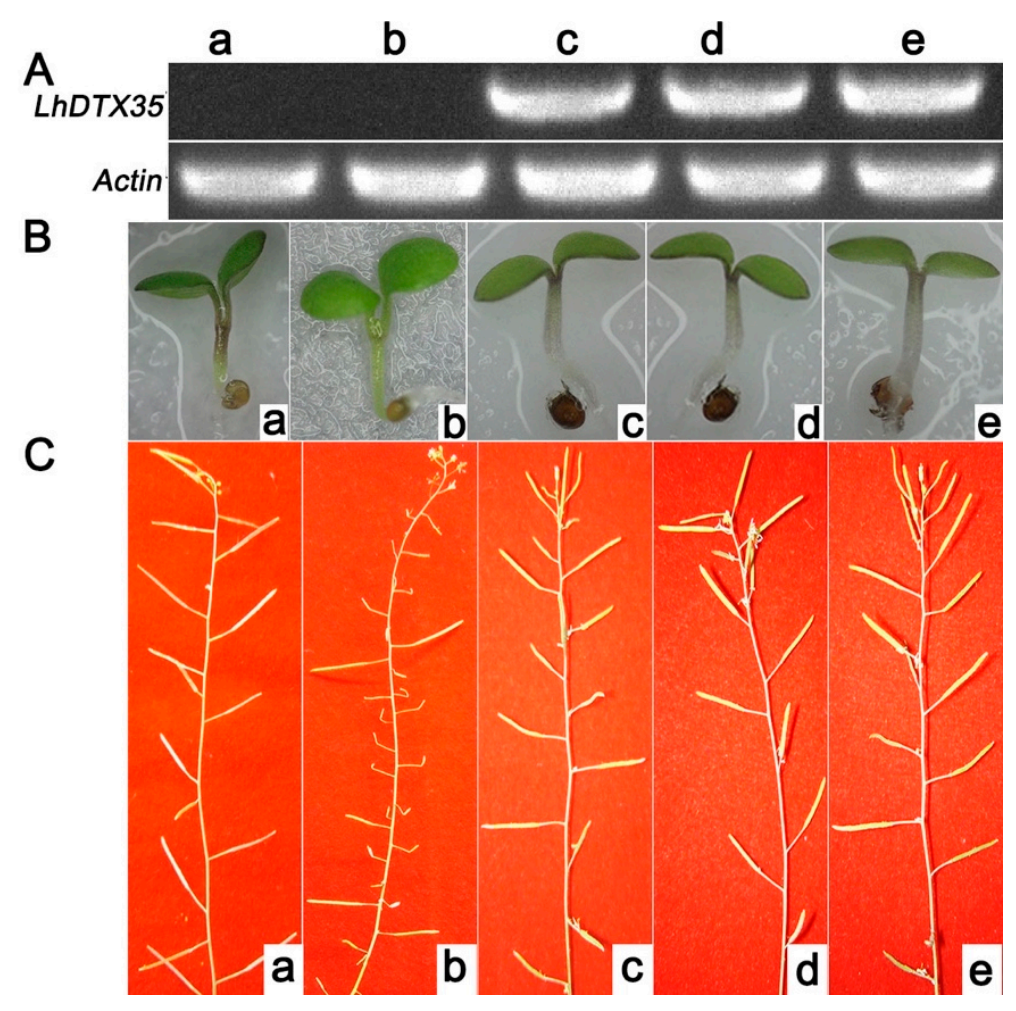

Figure 9. Functional complementation of the Arabidopsis DTX35 mutant with the LhDTX35 gene driven by the CaMV 35S promoter. (A) Expression of LhDTX35 in wild-type (a), mutant (b), and transgenic Arabidopsis seedlings (c-e). (B) Anthocyanin accumulation in wild-type (a), mutant (b), and transgenic Arabidopsis seedlings (c-e). (C) Silique fertility of wild-type (a), mutant (b), and transgenic Arabidopsis seedlings (c-e).

\section{Discussion}

Lilium has high ornamental value due to its wide variety of coloration patterns. Anthocyanins are important pigments responsible for Lilium flower pigmentation ranging from pink to purple. Therefore, the characterization of anthocyanins in Lilium is of considerable ongoing interest. In Lilium, the steps of anthocyanin biosynthesis and regulation are well established; however, the anthocyanin transportation mechanism remains unclear. In recent years, several transport proteins have been reported to be involved in anthocyanin transportation [9-11]. Among them, MATE is an important protein that transports anthocyanins. To date, whether the MATE protein is involved in anthocyanin transportation in Lilium is unknown.

In the present study, a flavonoid transport-related MATE candidate gene, LhDTX35, was cloned successfully based on our previous transcriptome data. Phylogenetic analysis revealed that the LhDTX35 gene clustered with DTX35 genes from Dendrobium catenatum, Phoenix dactylifera, and Oryza brachyantha (Figure 5). LhDTX35 is closely related to the DTX35 gene in Dendrobium catenatum, sharing $76.36 \%$ amino acid identity. Bioinformatics analysis revealed that the LhDTX35 gene contained sequences encoding the conserved domains typical of the MATE superfamily (Figure 3), suggesting that the protein encoded by LhDTX35 belonged to the MATE family. In addition, MATEs are important transport proteins that possess 12 typical transmembrane domains, and transmembrane structure prediction showed that LhDTX35 also has 12 typical transmembrane domains (Figure 4). These results indicated that LhDTX35 is a MATE protein in Lilium.

The expression profiles of anthocyanin biosynthetic genes and regulatory genes during anthocyanin accumulation at stages 1-4 were previously analyzed in 'Tiny Padhye', and the results showed that anthocyanin accumulation in tepals increased gradually at stages 1-4, reaching its highest level at stage 3 [21]. In this study, we revealed that the expression level of LhDTX35 was consistent with anthocyanin 
accumulation at stages 1-4 (Figure 7). Weak expression of LhDTX35 was detected in the tepals at stage 1 , when the anthocyanin content was lowest. In conjunction with the increasing anthocyanin content in the tepals, the transcript levels of LhDTX35 were gradually upregulated at stages 1-4, and both anthocyanin content and LhDTX35 gene transcript levels peaked at stage 3 (Figures 6 and 7). All the results indicated that LhDTX35 expression was positively correlated with anthocyanin accumulation in Lilium, suggesting that the LhDTX35 gene may be involved in the transport of anthocyanin in Lilium.

All the plant MATE proteins characterized to date have been localized to membranes [27]. To investigate whether LhDTX35 is involved in anthocyanin accumulation, its subcellular protein localization was examined. The results showed that LhDTX35 was localized to the membrane but not the cytoplasm, further suggesting that the LhDTX35 gene encoded a protein with the traits of MATE transport proteins. These results were also consistent with the results of the transmembrane structure prediction, that is, the protein encoded by LhDTX35 was predicted to have 12 typical transmembrane domains (Figure 4).

Complementation experiment is an effective method to analyze gene function because genetic transformation is a challenge for Lilium. Complementation has been used for this purpose in many species $[9,11,15]$. In this study, the silique-infertility phenotype of the DTX35 mutant was recovered when LhDTX35 was overexpressed in the DTX35 mutant of Arabidopsis, suggesting that the LhDTX35 gene has a similar function to that of DTX35 in Arabidopsis. The DTX35 gene is also called FFT (flower flavonoid transporter) [28]. The anthocyanin content of the FFT mutant was slightly less than that of the WT (Wile Type) in seedlings at one week of age [28]. Interestingly, the present study showed that the anthocyaninless phenotype of the DTX35 mutant was rescued by the complementation experiment (Figure 9B). These results suggested that the LhDTX35 gene was required for anthocyanin transportation in Lilium. MATE genes are part of a gene superfamily, including 58 MATEs in Arabidopsis and 53 MATEs in rice [29]. In the present study, only one MATE gene was cloned successfully, and whether any other MATE genes are involved in anthocyanin transportation in Lilium should be further studied.

\section{Conclusions}

In conclusion, we first identified a flavonoid transport-related MATE candidate gene based on transcriptome data in Lilium 'Tiny Padhye' and found that this gene shared high similarity with published DTX35 genes. Bioinformatics and phylogenetic analysis revealed that LhDTX35 belongs to the MATE gene family. Expression profiling showed that the expression of LhDTX35 was positively correlated with anthocyanin accumulation in the tepals of 'Tiny Padhye'. Subcellular localization results showed that the LhDTX35-encoded protein was localized to the membrane, suggesting that the protein encoded by the LhDTX35 gene possessed the traits of a MATE transporter. Silique-infertility and anthocyaninless hypocotyl phenotypes were recovered after the complementation experiment, indicating that LhDTX35 has a similar function to that of the DTX35 gene in Arabidopsis and is involved in anthocyanin transportation in Lilium. Our results provide insight into the mechanism of anthocyanin transportation in Lilium.

Author Contributions: Conceptualization, J.M. and L.X.; methodology, H.X.; investigation, P.Y., Y.T., Y.C., and G.H.; writing - original draft preparation, H.X.; writing-review and editing, L.X. and J.M.; funding acquisition, J.M. and H.X. All authors have read and agreed to the published version of the manuscript.

Funding: This research was funded by the National Natural Science Foundation of China, grant number 31672196, 31902048, and this research was also funded by the Fundamental Research Funds for Central Non-profit Scientific Institutions and the Science and Technology Innovation Program of the Chinese Academy of Agricultural Sciences.

Acknowledgments: We are grateful to Haiwen Zhang (Beijing Academy of Agricultural and Forestry sciences) for kindly offering Arabidopsis DTX35 mutant.

Conflicts of Interest: The authors declare no conflict of interest. 


\section{References}

1. Jeknić, Z.; Morré, J.T.; Jeknić, S.; Jevremović, S.; Subotić, A.; Chen, T.H.H. Cloning and Functional Characterization of a Gene for Capsanthin-Capsorubin Synthase from Tiger Lily (Lilium lancifolium Thunb. 'Splendens'). Plant Cell Physiol. 2012, 53, 1899-1912. [CrossRef] [PubMed]

2. Lai, Y.S.; Shimoyamada, Y.; Nakayama, M.; Yamagishi, M. Pigment accumulation and transcription of LhMYB12 and anthocyanin biosynthesis genes during flower development in the Asiatic hybrid lily (Lilium spp.). Plant Sci. 2012, 193, 136-147. [CrossRef] [PubMed]

3. Yamagishi, M.; Kishimoto, S.; Nakayama, M. Carotenoid composition and changes in expression of carotenoid biosynthetic genes in tepals of Asiatic hybrid lily. Plant Breed. 2010, 129, 100-107. [CrossRef]

4. Yamagishi, M.; Yoshida, Y.; Nakayama, M. The transcription factor LhMYB12 determines anthocyanin pigmentation in the tepals of Asiatic hybrid lilies (Lilium spp.) and regulates pigment quantity. Mol. Breed. 2012, 30, 913-925. [CrossRef]

5. Winkel-Shirley, B. Flavonoid biosynthesis. A colorful model for genetics, biochemistry, cell biology, and biotechnology. Plant Physiol. 2001, 126, 485-493. [CrossRef]

6. Zhao, J.; Dixon, R.A. The 'ins' and 'outs' of flavonoid transport. Trends Plant Sci. 2010, 15, 72-80. [CrossRef]

7. Holton, T.A.; Cornish, E.C. Genetics and biochemistry of anthocyanin biosynthesis. Plant Cell 1995, 7, 1071. [CrossRef]

8. Liu, Y.; Tikunov, Y.; Schouten, R.E.; Marcelis, L.F.; Visser, R.G.; Bovy, A. Anthocyanin biosynthesis and degradation mechanisms in Solanaceous vegetables: A review. Front. Chem. 2018, 6, 52. [CrossRef]

9. Hu, B.; Zhao, J.; Lai, B.; Qin, Y.; Wang, H.; Hu, G. LcGST4 is an anthocyanin-related glutathioneS-transferase gene in Litchi chinensis Sonn. Plant Cell Rep. 2016, 35, 831-843. [CrossRef]

10. Hu, D.G.; Sun, C.H.; Ma, Q.J.; You, C.X.; Cheng, L.; Hao, Y.J. MdMYB1 regulates anthocyanin and malate accumulation by directly facilitating their transport into vacuoles in apples. Plant Physiol. 2016, 170, 1315-1330. [CrossRef]

11. Luo, H.; Dai, C.; Li, Y.; Feng, J.; Liu, Z.; Kang, C. Reduced Anthocyanins in Petioles codes for a GST anthocyanin transporter that is essential for the foliage and fruit coloration in strawberry. J. Exp. Bot. 2018, 69, 2595-2608. [CrossRef] [PubMed]

12. Takanashi, K.; Shitan, N.; Yazaki, K. The multidrug and toxic compound extrusion (MATE) family in plants. Plant Biotechnol. 2014, 31, 417-430. [CrossRef]

13. Debeaujon, I.; Peeters, A.J.M.; Léon-Kloosterziel, K.M.; Koornneef, M. The TRANSPARENT TESTA12 gene of Arabidopsis encodes a multidrug secondary transporter-like protein required for flavonoid sequestration in vacuoles of the seed coat endothelium. Plant Cell 2001, 13, 853. [CrossRef] [PubMed]

14. Chen, L.; Liu, Y.; Liu, H.; Kang, L.; Geng, J.; Gai, Y.; Ding, Y.; Sun, H.; Li, Y. Identification and expression analysis of MATE genes involved in flavonoid transport in blueberry Plants. PLOS ONE 2015, 10, e0118578. [CrossRef] [PubMed]

15. Frank, S.; Keck, M.; Sagasser, M.; Niehaus, K.; Weisshaar, B.; Stracke, R. Two differentially expressed MATE factor genes from apple complement the Arabidopsis transparent testa12 mutant. Plant Biol. 2011, 13, 42-50. [CrossRef] [PubMed]

16. Gomez, C.; Terrier, N.; Torregrosa, L.; Vialet, S.; Fournier-Level, A.; Verriès, C.; Souquet, J.M.; Mazauric, J.P.; Klein, M.; Cheynier, V.; et al. Grapevine MATE-type proteins act as vacuolar H+-dependent acylated anthocyanin transporters. Plant Physiol. 2009, 150, 402. [CrossRef]

17. Xu, L.; Shen, Z.-L.; Chen, W.; Si, G.Y.; Meng, Y.; Guo, N.; Sun, X.; Cai, Y.P.; Lin, Y.; Gao, J.S. Phylogenetic analysis of upland cotton MATE gene family reveals a conserved subfamily involved in transport of proanthocyanidins. Mol. Biol. Rep. 2019, 46, 161-175. [CrossRef]

18. Liu, Y.; Lou, Q.; Xu, W.; Xin, Y.; Bassett, C.; Wang, Y. Characterization of a chalcone synthase $(C H S)$ flower-specific promoter from Lilium orential 'Sorbonne'. Plant Cell Rep. 2011, 30, 2187-2194. [CrossRef]

19. Nakatsuka, A.; Izumi, Y.; Yamagishi, M. Spatial and temporal expression of chalcone synthase and dihydroflavonol 4-reductase genes in the Asiatic hybrid lily. Plant Sci. 2003, 165, 759-767. [CrossRef]

20. Yamagishi, M. Oriental hybrid lily Sorbonne homologue of LhMYB12 regulates anthocyanin biosyntheses in flower tepals and tepal spots. Mol. Breed. 2011, 28, 381-389. [CrossRef] 
21. Xu, L.; Yang, P.; Feng, Y.; Xu, H.; Cao, Y.; Tang, Y.; Yuan, S.; Liu, X.; Ming, J. Spatiotemporal transcriptome analysis provides insights into bicolor tepal development in Lilium “Tiny Padhye". Front. Plant Sci. 2017, 8, 398. [CrossRef] [PubMed]

22. Trivedi, U.; Kaushik, S.; Kunjadia, P.; Matheshwaran, S.; Nareshkumar, G. Expression and purification of functional Anabaena PCC 7120 XisA protein. Protein Expr. Purif. 2015, 118, 64-69. [CrossRef] [PubMed]

23. Krogh, A.; Larsson, B.; Heijne, G.V.; Sonnhammer, E.L.L. Predicting transmembrane protein topology with a hidden markov model: Application to complete genomes. J. Mol. Biol. 2001, 305, 567-580. [CrossRef] [PubMed]

24. Livak, K.J.; Schmittgen, T.D. Analysis of relative gene expression data using real-time quantitative PCR and the $2^{-\Delta \Delta C T}$ method. Methods 2001, 25, 402-408. [CrossRef] [PubMed]

25. Jyothishwaran, G.; Kotresha, D.; Selvaraj, T.; Srideshikan, S.H.; Rajvanshi, P.K.; Jayabaskaran, C. A modified freeze-thaw method for efficient transformation of Agrobacterium tumefaciens. Curr. Sci. Indian 2007, 6, 770-772.

26. Clough, S.J.; Bent, A.F. Floral dip: A simplified method for Agrobacterium-mediated transformation of Arabidopsis thaliana. Plant J. 1998, 16, 735-743. [CrossRef]

27. Eckardt, N.A. Move it on out with MATEs. Plant Cell 2001, 13, 1477-1480. [CrossRef]

28. Thompson, E.P.; Wilkins, C.; Demidchik, V.; Davies, J.M.; Glover, B.J. An Arabidopsis flavonoid transporter is required for anther dehiscence and pollen development. J. Exp. Bot. 2009, 61, 439-451. [CrossRef]

29. Tiwari, M.; Sharma, D.; Singh, M.; Tripathi, R.D.; Trivedi, P.K. Expression of OsMATE1 and OsMATE2 alters development, stress responses and pathogen susceptibility in Arabidopsis. Sci. Rep. 2014, 4, 3964. [CrossRef]

(C) 2020 by the authors. Licensee MDPI, Basel, Switzerland. This article is an open access article distributed under the terms and conditions of the Creative Commons Attribution (CC BY) license (http://creativecommons.org/licenses/by/4.0/). 